\title{
UNIT DISTANCE GRAPHS AND ALGEBRAIC INTEGERS
}

\author{
DANYLO RADCHENKO
}

\begin{abstract}
We answer a question of Brass about vertex degrees in unit distance graphs of finitely generated additive subgroups of $\mathbb{R}^{2}$.
\end{abstract}

The following problem was posed in [2, p. 186] (see also [1]).

Problem 1. Does there exist a finitely generated additive subgroup $\mathcal{A} \subset \mathbb{R}^{2}$ such that there are infinitely many elements of $\mathcal{A}$ lying on the unit circle?

If we look at the graph whose vertex set is a given finitely generated additive group $\mathcal{A} \subset$ $\mathbb{R}^{2}$, and where $x, y \in \mathcal{A}$ are joined by an edge if and only if $|x-y|=1$, then the question is whether such a graph can have infinite vertex degrees. This question was motivated by the problem of Erdös about maximal number of unit distances among $n$ points in the plane [3]. In this short note we give a positive answer to Problem 1. Let us denote

$$
\left\langle v_{1}, \ldots, v_{n}\right\rangle_{\mathbb{Z}}:=\left\{a_{1} v_{1}+\cdots+a_{n} v_{n} \mid a_{1}, \ldots, a_{n} \in \mathbb{Z}\right\} .
$$

Theorem 1. There exist four vectors $v_{1}, v_{2}, v_{3}, v_{4} \in \mathbb{R}^{2}$ such that $\mathcal{A}=\left\langle v_{1}, v_{2}, v_{3}, v_{4}\right\rangle_{\mathbb{Z}}$ has infinitely many elements on the unit circle.

Proof. We identify $\mathbb{R}^{2}$ with $\mathbb{C}$. Let $p \in \mathbb{Z}[z]$ be the irreducible polynomial

$$
p(z)=z^{4}-z^{3}-z^{2}-z+1 .
$$

Using Descartes' rule of sign and noting that $p(1)<0$ we see that $p$ has exactly two positive real roots. Since $z^{4}-z^{2}+1>0$ for all $z \in \mathbb{R}$, it has no negative roots. Since $p$ is reciprocal, the two remaining complex roots $\alpha$ and $\bar{\alpha}$ must be inverses of each other, so that $|\alpha|=1$. Next, we have $\alpha^{m} \in\left\langle 1, \alpha, \alpha^{2}, \alpha^{3}\right\rangle_{\mathbb{Z}}$ for all $m \in \mathbb{Z}$ because $p$ is monic and has integer coefficients. Moreover, the numbers $\alpha^{m}, m \in \mathbb{Z}$ satisfy $\left|\alpha^{m}\right|=1$ and are all distinct since $p$ is not divisible by any cyclotomic polynomial. Therefore, the set $\mathcal{A}=\left\langle 1, \alpha, \alpha^{2}, \alpha^{3}\right\rangle_{\mathbb{Z}}$ has infinitely many elements on the unit circle.

Of course, the above construction works for any algebraic integer $\alpha \in \mathbb{C}$ with $|\alpha|=1$. If we take $\alpha$ to be a primitive $m$-th root of unity, then the resulting unit distance graph has degree $m$ or $2 m$ depending on the parity of $m$. Taking $\alpha \in \mathbb{C}$ to be any other algebraic integer of absolute value 1 gives a unit distance graph of infinite degree. For example, one can take any non-real conjugate of a Salem number (a positive algebraic integer, all of whose conjugates have absolute value $\leq 1$, see [4, Ch. 3]); this is exactly what was done in the proof above.

It is also natural to ask what is the minimal possible number of generators for such $\mathcal{A}$. The following result shows that in this sense Theorem 1 is optimal.

Theorem 2. If an additive subgroup $\mathcal{A} \subset \mathbb{R}^{2}$ has rank $\leq 3$, then it has only finitely many elements on the unit circle $S^{1}$.

Proof. Suppose that $\mathcal{A} \cap S^{1}$ is infinite. We can restrict to the case when $\operatorname{rk}(\mathcal{A})=3$ and $\mathcal{A}$ spans $\mathbb{R}^{2}$ over reals, since otherwise $\mathcal{A}$ is contained in a lattice or in a line and thus intersects $S^{1}$ in a finite set. By the same argument, the subgroup generated by $\mathcal{A} \cap S^{1}$ must also have rank 3 , and therefore we can choose three elements $v_{1}, v_{2}, v_{3} \in \mathcal{A} \cap S^{1}$ and an integer $n>0$ such that $\mathcal{A} \subseteq\left\langle v_{1} / n, v_{2} / n, v_{3} / n\right\rangle_{\mathbb{Z}}$. 
Let us denote $\left(v_{1}, v_{2}\right)=\gamma,\left(v_{2}, v_{3}\right)=\alpha$, and $\left(v_{3}, v_{1}\right)=\beta$. Before we continue, let us record some elementary identities between $v_{i}$ and the inner products $\alpha, \beta, \gamma$. First, since the vectors $v_{i}$ are in $\mathbb{R}^{2}$, the determinant of the Gram matrix of $v_{1}, v_{2}, v_{3}$ is 0 , that is

$$
1+2 \alpha \beta \gamma-\alpha^{2}-\beta^{2}-\gamma^{2}=0 .
$$

Next, since $v_{i}$ are linearly independent over $\mathbb{Q}$, we have $|\alpha|,|\beta|,|\gamma|<1$, and thus, using (1), we see that $\alpha \neq \beta \gamma, \beta \neq \alpha \gamma$, and $\gamma \neq \alpha \beta$. Finally, we have the following identity

$$
\frac{v_{1}}{\alpha-\beta \gamma}+\frac{v_{2}}{\beta-\alpha \gamma}+\frac{v_{3}}{\gamma-\alpha \beta}=0 \text {. }
$$

Let $Q(x, y, z)=\left\|x v_{1}+y v_{2}+z v_{3}\right\|^{2}$ and let $\mathcal{S}$ be the set of solutions $(x, y, z) \in \mathbb{Z}^{3}$ of equation $Q(x, y, z)=n^{2}$; by our assumption this set is infinite. A triple $(x, y, z)$ belongs to $\mathcal{S}$ if and only if

$$
x^{2}+y^{2}+z^{2}+2 \alpha y z+2 \beta z x+2 \gamma x y=n^{2} .
$$

Since $v_{1}, v_{2}$, and $v_{3}$ are independent over $\mathbb{Q}$, the intersection of $\mathcal{S}$ with any plane in $\mathbb{Z}^{3}$ is finite. In particular, the set $\mathcal{S}_{0}=\{(x, y, z) \in \mathcal{S} \mid x y z \neq 0\}$ is infinite, and the condition for $(x, y, z) \in \mathcal{S}_{0}$ can be rewritten as

$$
\frac{\alpha}{x}+\frac{\beta}{y}+\frac{\gamma}{z}=\frac{n^{2}-x^{2}-y^{2}-z^{2}}{2 x y z} .
$$

Denote $\mathcal{V}=\left\{(1 / x, 1 / y, 1 / z) \mid(x, y, z) \in \mathcal{S}_{0}\right\}$. We consider three cases.

Case 1. The $\mathbb{Q}$-span of $\mathcal{V}$ is $\mathbb{Q}^{3}$. By choosing any three linearly independent vectors in $\mathcal{V}$ and solving (3) for $\alpha, \beta, \gamma$, we get that $\alpha, \beta$, and $\gamma$ must be rational. But then (2) gives a linear relation over $\mathbb{Q}$ contradicting the fact that $\operatorname{rk}(\mathcal{A})=3$.

Case 2. The $\mathbb{Q}$-span of $\mathcal{V}$ is 1 -dimensional. Then $\mathcal{S}_{0}$ is contained in a line through the origin, and by homogeneity of $Q$ we get that $\left|\mathcal{S}_{0}\right| \leq 2$, a contradiction.

Case 3. The $\mathbb{Q}$-span of $\mathcal{V}$ is 2-dimensional. Then there is a unique nonzero triple $(a, b, c) \in \mathbb{Z}^{3}$ such that for all $(x, y, z) \in \mathcal{S}_{0}$ we have $a / x+b / y+c / z=0$. In this case, by solving (3) for $\alpha, \beta, \gamma$, we get

$$
\alpha=\lambda a+\alpha_{0}, \quad \beta=\lambda b+\beta_{0}, \quad \gamma=\lambda c+\gamma_{0}
$$

for some $\left(\alpha_{0}, \beta_{0}, \gamma_{0}\right) \in \mathbb{Q}^{3}$ and $\lambda \in \mathbb{R}$. We may assume that $a b c \neq 0$, since otherwise $\mathcal{S}_{0}$ would be contained in one of the three planes $a y+b x=0, a z+c x=0$, or $b z+c y=0$, and hence would be finite.

Next, recall that all integer solutions of $1 / x+1 / y+1 / z=0$ are given by $x=d s t$, $y=d r t, z=d r s$ for some nonzero integers $d, r, s, t$ with $r+s+t=0$ (simply note that the equation is equivalent to $z^{2}=(z+x)(z+y)$ that is in turn equivalent to $\left.z^{2}=x^{\prime} y^{\prime}\right)$. By applying this observation to the equation $a / x+b / y+c / z=0$ we deduce that any solution $(x, y, z) \in \mathcal{S}_{0}$ can be written as

$$
x=\frac{d s t}{b c}, \quad y=\frac{d r t}{a c}, \quad z=\frac{d r s}{a b}
$$

for some nonzero integers $d, r, s, t$ with $r+s+t=0$. Plugging these expressions back into $Q$ and setting $v_{3}=\kappa_{1} v_{1}+\kappa_{2} v_{2}$ (where $\kappa_{i}$ can be computed from (2)) we get

$$
n^{2}=\left\|x v_{1}+y v_{2}+z v_{3}\right\|^{2}=\frac{d^{2}}{(a b c)^{2}}\left\|s\left(\left(\kappa_{1} c-a\right) r-a s\right) v_{1}+r\left(\left(\kappa_{2} c-b\right) s-b r\right) v_{2}\right\|^{2},
$$

and thus, setting $\varphi_{1}(r, s)=\left(\kappa_{1} c-a\right) r-a s$ and $\varphi_{2}(r, s)=\left(\kappa_{2} c-b\right) s-b r$, we get

$$
\left\|s \varphi_{1}(r, s) v_{1}+r \varphi_{2}(r, s) v_{2}\right\|^{2}=\frac{(a b c n)^{2}}{d^{2}} \leq(a b c n)^{2} .
$$


Since $v_{1}$ and $v_{2}$ form a basis in $\mathbb{R}^{2}$ and $|s|,|r| \geq 1$ we get that $\left|\varphi_{i}(r, s)\right| \leq C$ for some constant $C$ that depends only on $a, b, c, n$, and $\gamma$. If $\varphi_{1}$ and $\varphi_{2}$ are not proportional, then these two inequalities define a bounded region in the $(r, s)$-plane, and hence there are only finitely many solutions in this case, contrary to our assumption. Therefore, $\varphi_{1}$ and $\varphi_{2}$ must be proportional, that is

$$
\left(\kappa_{1} c-a\right)\left(\kappa_{2} c-b\right)-a b=0
$$

or equivalently

$$
a(\alpha-\beta \gamma)+b(\beta-\alpha \gamma)+c(\gamma-\alpha \beta)=0 .
$$

If we now substitute (4) into (1) and into (5), we obtain two different polynomial equations on $\lambda, p_{1}(\lambda)=0$ and $p_{2}(\lambda)=0$, where

$$
\begin{aligned}
p_{1}(\lambda) & =1+2 \alpha \beta \gamma-\alpha^{2}-\beta^{2}-\gamma^{2}=2 a b c \lambda^{3}+\left(2 b c \alpha_{0}+2 a c \beta_{0}+2 a b \gamma_{0}-a^{2}-b^{2}-c^{2}\right) \lambda^{2} \\
& +2\left(a \beta_{0} \gamma_{0}+b \alpha_{0} \gamma_{0}+c \alpha_{0} \beta_{0}-a \alpha_{0}-b \beta_{0}-c \gamma_{0}\right) \lambda+\left(2+\alpha_{0} \beta_{0} \gamma_{0}-\alpha_{0}^{2}-\beta_{0}^{2}-\gamma_{0}^{2}\right)
\end{aligned}
$$

and

$$
\begin{aligned}
& p_{2}(\lambda)=a(\alpha-\beta \gamma)+b(\beta-\alpha \gamma)+c(\gamma-\alpha \beta)=-3 a b c \lambda^{2} \\
& -\left(2 b c \alpha_{0}+2 a c \beta_{0}+2 a b \gamma_{0}-a^{2}-b^{2}-c^{2}\right) \lambda-\left(a \beta_{0} \gamma_{0}+b \alpha_{0} \gamma_{0}+c \alpha_{0} \beta_{0}-a \alpha_{0}-b \beta_{0}-c \gamma_{0}\right) .
\end{aligned}
$$

We observe that $2 p_{2}(\lambda)=-p_{1}^{\prime}(\lambda)$, so that $\lambda$ is a double root of a cubic polynomial $p_{1}$ with rational coefficients. It is well-known that this implies $\lambda \in \mathbb{Q}$, but then $\alpha, \beta$, and $\gamma$ are also rational, and once again we arrive at a contradiction.

\section{REFERENCES}

[1] P. Brass, Erdös's distance problems in normed spaces, Computational Geometry 6 (4), pp. 195-214 (1996).

[2] P. Brass, W. Moser, J. Pach, Research Problems in Discrete Geometry, Springer, Berlin, 2005.

[3] P. Erdős, On sets of distances of n points, Amer. Math. Monthly 53, pp. 248-250 (1946).

[4] R. Salem, Algebraic numbers and Fourier analysis, Heath mathematical monographs, Heath, 1963.

Max Planck Institute for Mathematics, Vivatsgasse 7, 53111 Bonn, Germany

E-mail address: danradchenko@gmail.com 